\title{
Comparison of integrated and composed step-down converter in terms of EMC
}

\author{
Peter Janků ${ }^{1, *}$, Luboš Lorenc ${ }^{1}$ and Tomáš Dulík ${ }^{1}$ \\ ${ }^{1}$ Tomas Bata University in Zlin, Faculty of applied informatics, Nad Stranemi 4511, 76005 Zlin
}

\begin{abstract}
Stabilized voltage sources, even linear or working on a switching principle, are an integral part of almost all electronic devices. Hand in hand with the pressure of environmental protection, there comes an emphasis on reducing energy consumption, resulting in preference of switching solutions. On the other hand, considering the issues on electromagnetic compatibility, each switching-mode operated power source produces considerable amount of interferences. In the framework of the hereby described experiment, the authors of the paper tested several voltage sources operating on the switching principle. This paper provides a description of the experiment and the obtained results.
\end{abstract}

\section{Introduction}

In a modern world, different electronic devices are unnecessary parts of a daily live. Moreover, the rising popularity of IoT devices together with a pressure on the target device size and power efficiency, it creates new challenges for a HW developers. At the opposite side of these situation the modern designs have to pass really strict EMC limitations and standards.

Mostly every electronic device has to have inbuilt power source in form of a linear stabilizer or a voltage converter. These parts of designs are usually one of the most important tasks in a HW design work. They are usually supplying highest currents in the device circuit and they could have a really significant impact on a final EMC characteristic. Therefore, the selection and construction of a suitable power source is really tricky. $[4,5]$

In this paper we will deal with step-down voltage converters which are in terms of EMC usually more problematic then linear stabilizers. For the HW developers there are two possibilities how to construct a power source based on step-down voltage converter. The first one is to compose it from different discrete and integrated parts (composed step-down converter), the second one is to use it as an embeddable integrated device (integrated step-down converter) These research free follows previous presented studies $[1,2,3]$ in which different step down converters were discussed with special attention on their EMC compatibility. The measurements of real characteristics of integrated stepdown converter are discussed in this paper together with comparison with previous published composed stepdown converters. All measurements and measures were performed to meet the requirements placed by working conditions and the standard EN 61000-6-3. [2, 3, 4, 5]

\section{Description of used converters}

In this paper the comparison between integrated and composed step down converters is discussed. The Traco TSR-1-2450 [7] was selected as an "integrated stepdown converter" and the circuit based on AOZ1284 [6], which was published in previous research was selected as a "composed step-down converter". Both of them has nearly the same characteristic.

\subsection{Converter parameters}

Table 1. Electrical parameters of step-down converters

\begin{tabular}{|c|c|c|}
\hline Parameter & AOZ1284 & TSR-1-2450 \\
\hline Maximal input voltage & $30 \mathrm{~V}$ & $36 \mathrm{~V}$ \\
\hline Output voltage & $5 \mathrm{~V}$ & $5 \mathrm{~V}$ \\
\hline Maximal output current & $800 \mathrm{~mA}$ & $1 \mathrm{~A}$ \\
\hline Min. power effeciency & $80 \%$ & $84 \%$ \\
\hline
\end{tabular}

As could be seen in Table 1, both converters has nearly the same electrical characteristics. Therefore we can simply compare their real electrical and EMC characteristics. For all measurements, the reference input voltage level was set at $12 \mathrm{~V}$ and $24 \mathrm{~V}$.

\footnotetext{
* Corresponding author: janku@,fai.utb.cz
} 


\subsection{Integrated voltage converter TSR-1-2450}

The selected integrated converter is an electronic device, which has integrated all necessary parts for work as nonisolated DC/DC converter. It has wide range of input voltage level $(6,5 \mathrm{~V}$ to $36 \mathrm{~V})$ and high efficiency (max. $96 \%$ ). Moreover, the output current can achieved 1 A level (5W output). It is inbuilt in package, which can directly replace standard linear stabilizers with package TO-220. The picture of this device could be seen at Fig. 1.

As stated before, the input/output filtering capacitors are inbuilt as well as other necessary parts; therefore, the usage of this part is really easy. For normal operation connection of one simple input capacitor is recommended by manufacturer. In order to accomplish international EMC standard EN 55022, it is recommended to connect simple LC filter at the input of this device. [7]

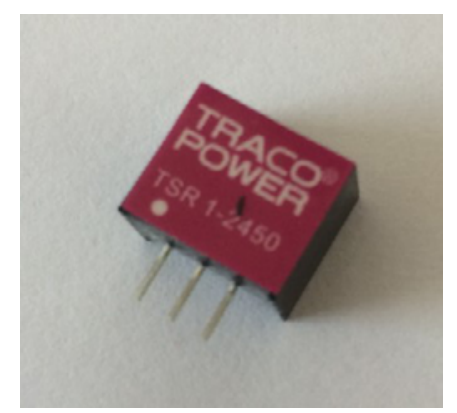

Fig. 1. Integrated step-down converter TSR-1-2450.

\subsection{Composed step-down converter based on AOZ1284}

As a second device for this comparison the step-down converter composed from passive parts and based on AOZ1284 was chosen. Its construction and detailed characteristics were published in the previous paper [2].

The whole circuit is based on a previous mentioned integrated device complemented by set of passive electronic parts. The main current loop consisting of inductor, switching N-MOSFET transistor and Schottky diode are placed outside of IO. Moreover, other capacitors are used for setting the converter's working frequency, for stabilizing input and output voltage level and for feedback compensation. The feedback resistors are also place outside of the IO. These facts means, that the electronic circuit of this step-down converter is significantly more complex than in previous case. At the other hand, the developer is able to tune more parameters of power source in order to fit specific characteristics of supplied device. [12, 13]

\section{Measurements and results}

The described converters were constructed and/or prepared as a functional sample and tested for achieving of the request parameters. Consequently, the sets of measurements were performed in order to gain data set needed for implementation of these converters into final products.

Since the target use of circuits was not specified, the standard EN 61000-6-3 had been chosen as a base requirement for EMC tests.

The predefined tests were as follows:

- output voltage stability vs. output load,

- power efficiency at different output loads and input voltages,

- interference currents on the input cables measured by LISN ENV216,

- electromagnetic interference without a cover according to EN 61000-6-3.

\subsection{Measurement configurations}

The predefined sets of measurements from previous chapter were performed at three different measurement networks:

- Network configuration for measurement DC characteristics,

- Network configuration for measurement of interferences on input wires,

- Network configuration for measurement of radiated electromagnetic field.

\subsubsection{Network for measurement of $D C$ characteristic}

The first measurements of DC characteristics were performed at a network composed from the laboratory power source Velleman PS3010, the measured converter and the programmable load Array 3721A. As is depicted in Fig. 2 the input voltages and currents were measured by using two true RMS multimeters connected directly at controlled converter's input. The precise output values were measure directly by programmable load.

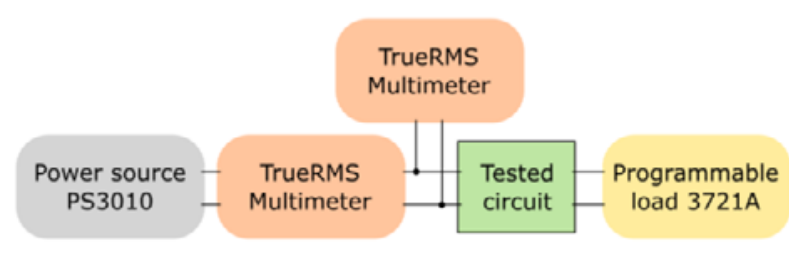

Fig. 2. Measurement network for comparison of DC characteristics

\subsubsection{Radiated electromagnetic field}

The measurement network used for measurement of radiated electromagnetic field is visualized in Fig. 3. As can be seen, measured sample was placed in a semi anechoic chamber which limits the signals naturally presented in environment.

The measurement network was composed of a bilogarithmical antenna Teseq Bilog CBL 6112 
connected through interconnection network to the Rohde\&Schvartz ESU 8 test receiver and spectral analyser. Data obtained by ESU 8 were processed by using EMC32 software.

Because of measurement need to be performed in different working conditions, the measured sample were connected to the laboratory power source and loaded by programmable load. $[9,10,11]$

In order to eliminate of electromagnetic field emitted by power source and programmable load, the last measurement was performed with bypassed measured sample.

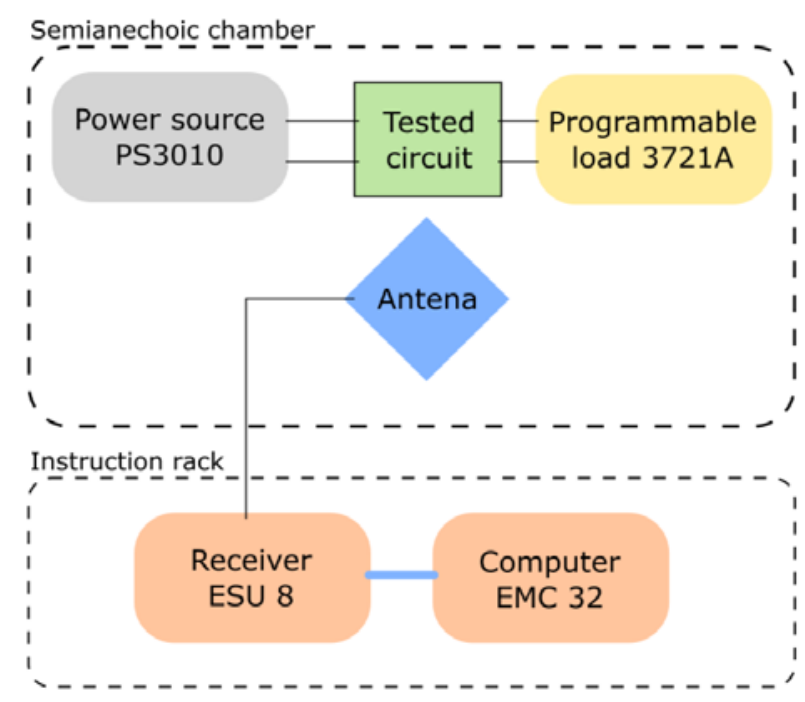

Fig. 4. Measurement network used for measure of radiate electromagnetic field.

\subsubsection{Interferences on input wires}

The measurement of interferences on input wires was performed in the standard combination used in previous cases. The measured sample is powered by using laboratory power source and it is connected to the programmable loads set in continuous current mode. Moreover, the LISN Rohde \& Schwarz ENV216 was connected between power source and measured sample. This device can meet the requirements of CISPR 16-1-2, EN 55016-1-2. Because of performed measurements were not final (measured converters are usually only one part of final device) the current probe could be used as in previous researches [2].

The obtained data were collected in EMC 32 software and post procced by using MS Excel and Wolfram Mathematica.

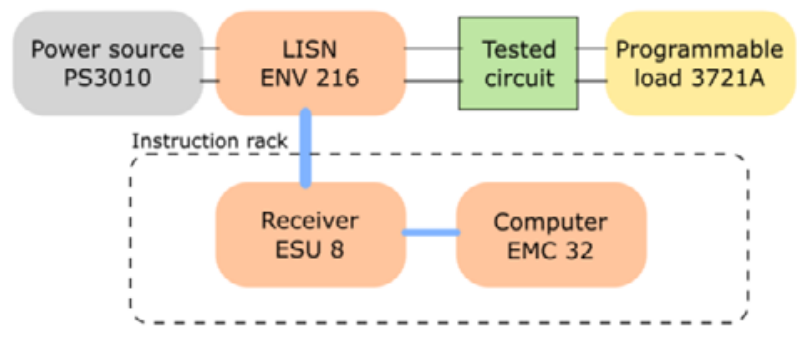

Fig. 3. Configuration of measurement network for measure of interferences on input wires.

\subsection{Results}

The measurement networks descripted in previous chapters were used in order to obtain real data. Both observed step-down converters were placed into same working conditions and same values were checked.

\subsubsection{DC parameters}

As was told before, compared step-down converters were constructed with nearly same output parameters. By using the DC measure network, described in the section 3.1.1, these parameters and its characteristics were measured. These measurements were done in two steps. At first time the converters efficiency was checked and at second the stability of output voltage was compared.

The graphs of converters efficiency in different working conditions are depicted in fig. 5. As could be seen, both of converters are able to achieve nearly the same efficiency. The composed step-down converter based on AOZ 1284 has the lower level of efficiency (78\%) with $12 \mathrm{~V}$ input and $800 \mathrm{~mA}$ output load. This point is probably caused by limitation of some selected parts. At the other hand, integrated converter Traco achieved even lower level of efficiency $-76 \%$. It has to be notice that this result is lower than typical values provided by manufacturer's datasheet.

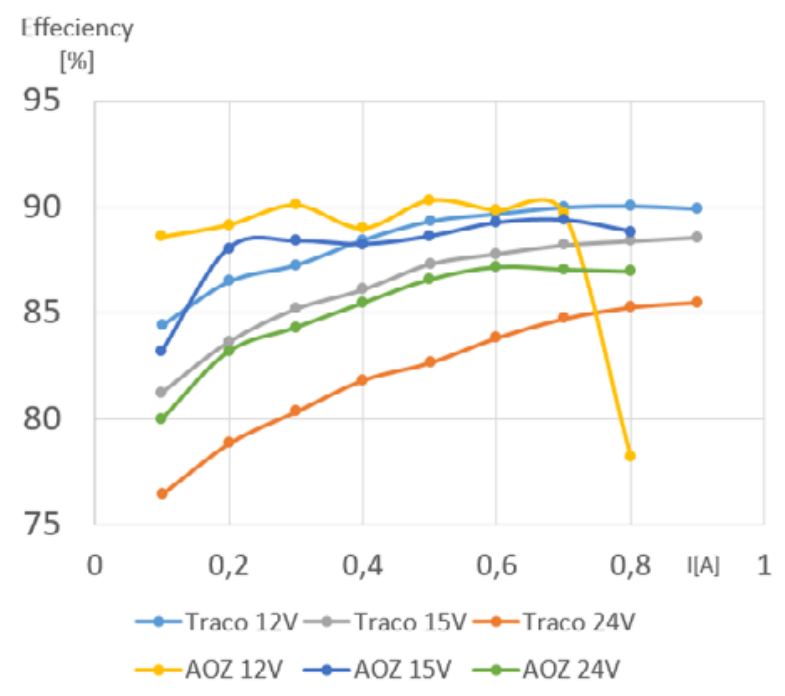

Fig. 5. Comparison of converters efficiency in different working conditions. 
The dependencies of output voltage level on output load are shown in Fig. 6. As can be seen the maximum difference in output voltage for step-down converter Traco is $35 \mathrm{mV}$ and for composed converter AOZ is 44 $\mathrm{mV}$. Moreover, the average value of output voltage level is lower in AOZ converter then Traco. This could be problem for some installation; at the other hand, in case of composed converter this parameter could be finetuned by of resistors in feedback divider.

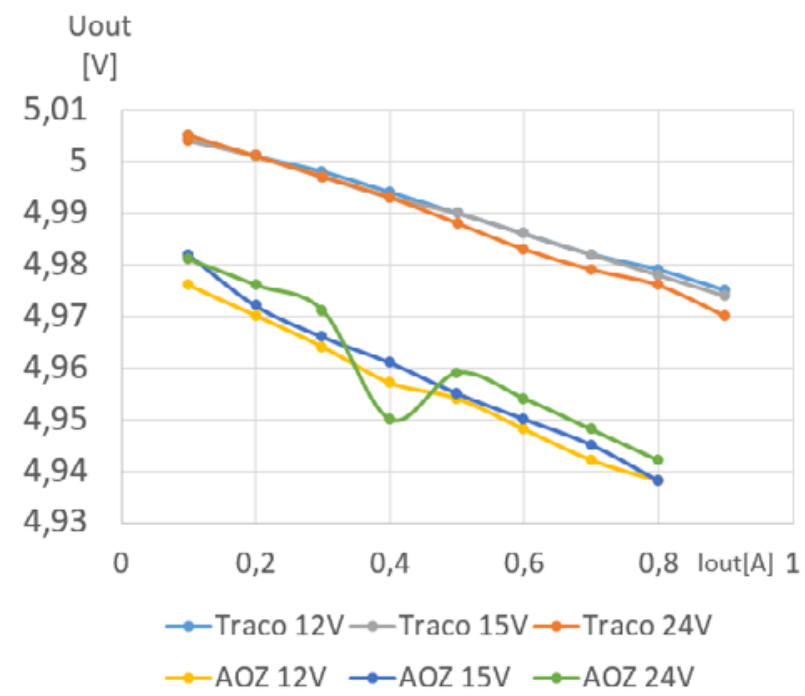

Fig. 6. Output voltage dependence on output current measured with different level of input voltage.

\subsubsection{Radiated electromagnetic field}

As it was mentioned in previous sections, the measurement of radiated electromagnetic field was one part of this comparison. The measurement method and base bade was used according to standard EN 61000-6-3. The base bend was used in rang of $30 \mathrm{MHz}$ to $1 \mathrm{GHz}$.

One of the worst measured outputs can be seen in Fig. 7. This graph describes the level of radiated electromagnetic field for integrated converter Traco. Even if there is a problematic point around the $180 \mathrm{MHz}$, these don't have to be strictly problem - the radiated field is strictly depend on cases in which is measured. This means, that for our purposes we measured just only converter without any cover or case. In real usage this converts will be probably inbuilt in device placed in cover, box or case.

The results of a corresponding measurement performed at composed step-down converter AOZ could be seen in previous the paper [2]. There was the same case - one problematic point but measurement without case.

\subsubsection{Interferences on input wires}

According to a principle of operation of the step down converters, the current ripples and/or voltage peaks at input wires are expected. The set of measurements required by standard EN $61000-6-3$ is really large. Therefore, due to size limit of this paper, only two measurements at positive input wire are presented. As was told before, these measurements were performed by using measure network defined in standard EN 61000-63. The base of this network was ENV 2016 LSN.

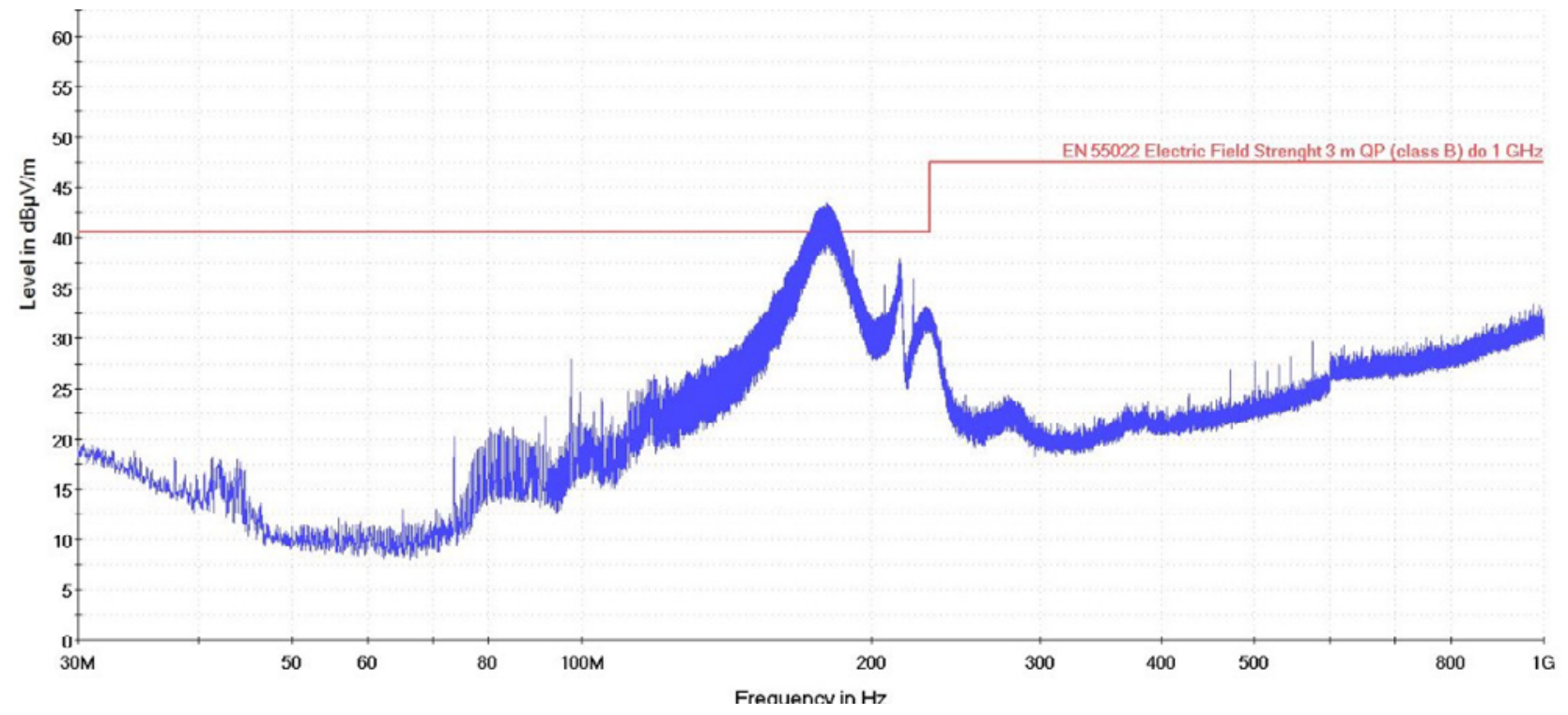

Fig. 6. Output voltage dependence on output current measured with different level of input voltage. 


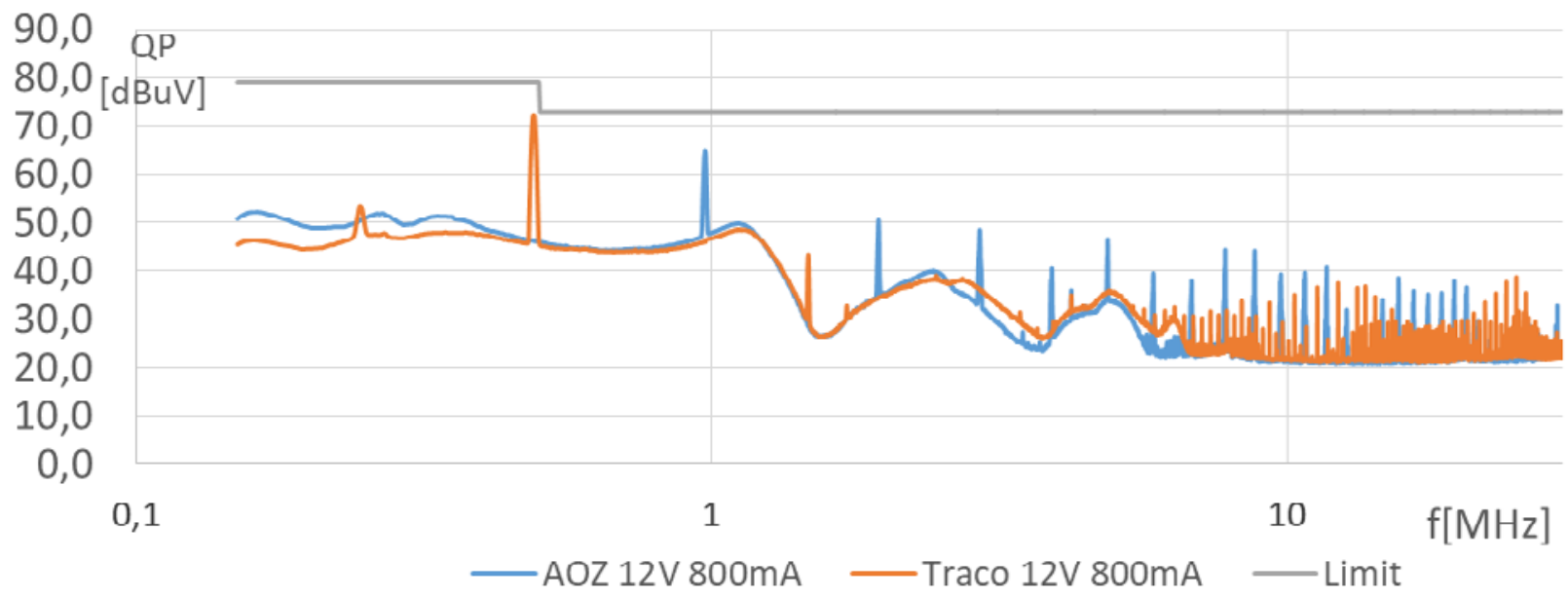

Fig. 7. Output voltage dependence on output current measured with different level of input voltage.

The comparison of spectrums of interferences on input wires can be seen in Fig. 7 and Fig. 8. Both this measurements were performed with maximum output load set at level of $800 \mathrm{~mA}$. The fig. 7 depicted the state, when the 12 volts are used as an input into converters. The measurement was performed in frequency range from $150 \mathrm{kHz}$ to $30 \mathrm{MHz}$.

For both converters, the first peak is measured at the converters working frequency - for $\mathrm{AOZ}$ it is $961 \mathrm{kHz}$, while for Traco it is $580 \mathrm{kHz}$. The next peaks mostly correspond with harmonic multiplies of working frequency. Even though the peaks, both of converters are able to full-fill the limit define by standard. It has to notice, that these measurements were performed with power filters included into input wires. The characteristic of filter used for AOZ step down converter was described in previous research. The filter used together with Traco converter corresponds to recommendations defined in manufacture datasheet.

The results of second measurement is shown in Fig. 8. This measurement was performed in the same configuration as the previous one, but the input voltage was changed to $24 \mathrm{~V}$. The position of frequency peaks and it's level is nearly the same as in the previous case. Even if there is a lot of peaks, no one exceeds the limit defined by the standard.

All performed measurements were done by using Quasi-peak detector. In fact, the standard defines the same set measurements performed by using average detector with different limits. Both of them has to pass. Due to limit size of this paper, only QP measurements are discussed. Moreover, the interferences on input wires could slightly depend on characteristic of load. This mean that in real case - in real device, the level of interferences could be different.

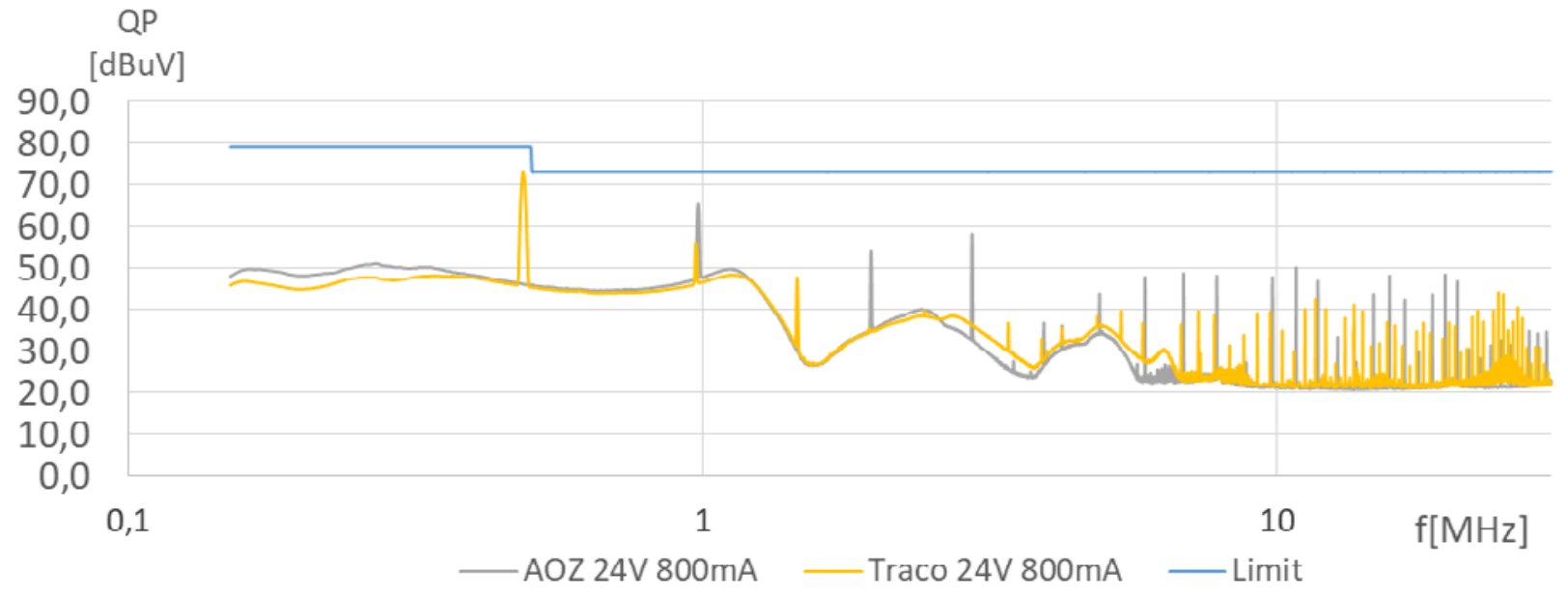

Fig. 8. Output voltage dependence on output current measured with different level of input voltage. 


\section{Conclusions}

This paper provides comparison of two small cheap stepdown converters. The converter composed from discreet parts and based on AOZ 1284 was chosen as the first one compared device. Its construction and basic characteristics were discussed in previous published paper. The integrated step-down converter Traco TSR-12450 was chosen as the second device. It is nonisolated fully integrated step down converter, which can be used as a replacement to a well-known linear stabilisers 78xx.

Both converters were construct/prepared as a functional sample and the same set of measurement were performed on them. The DC measurements agree that both converters has nearly the same characteristics and that they are able to full-fill requirements of different electronical devices. The radiated electromagnetic field measurement shown, that both of them are likely to meet the requirements defined by standard. The interferences on input wires were in both cases performed on devices with connected input filters. Thanks to this, no significant problems were founded when the quasi-peak detector was used.

According the data we obtained it can be suspected that both converters will be able to pass all requirements defined by standard EN61000-6-3 after small tuning of input filters.

Because of the voltage converter is usually only one part of electronic device and all its EMC characteristics depends on the connected load, performed measurements are only tentative. At the other hand, they can provide basic overview of device characteristics for electronic device designers.

As was described in previous text, the used standard defines significantly larger set of needed tests. These measurements was not performed due to limit size of this paper and they could be interesting subject of future research. Moreover, there is a set of isolating converters at the market with the nearly the same parameters which can be the subjects for future research.

\section{Acknowledgement}

This work was supported by the Ministry of Education, Youth and Sports of the Czech Republic within the National Sustainability Programme project No. LO1303 (MSMT7778/2014)

\section{References}

1. M. Pospisilik, M. Adamek, R. M. S. Silva, Different constructions of step down voltage converters in terms of $E M C$, International Journal of Circuits, Systems and Signal Processing, Volume 10, (2016), p. 190-199, ISSN: $1998-4464$

2. P. Janku, M. Pospisilik, T. Dulik, Construction of compact step down voltage converter in terms of EMC, MATEC Web Conf. 7601010 (2016),DOI: $10.1051 /$ matecconf/20167601010

\footnotetext{
* Corresponding author: janku@,fai.utb.cz
}

3. J. Svacina, Electromagnetic Compatibility [Elektromagneticka kompatibilita], Brno: VUT Brno, (2002).

4. C. R. Paul, Introduction to Electromagnetic Compatibility, New York: John Wiley, (1992).

5. P. A. Chatterton, M. A. Houlden, EMC Electromagnetic Theory to Practical Design, New York: John Wiley, (1991).

6. ALPHA \& OMEGA Semicondutor (2012, March), AOZ1284 Datasheet[online]. Available: http://aosmd.com/res/data_sheets/AOZ1284PI.pdf

7. Traco Power DC/DC Converters, TSR-12450. (2015, October) Datasheet [online] Available: http://www.tracopower.com/products/tsrn1.pdf

8. M. Pospisilik., T. Dulik, International Journal of Circuits, Systems and Signal Processing, 8, (2014), pp $518-528$

9. M. Mann, B. Gutheil, J. Zastrau, P. Weiss, "Electromagnetic field measurements - Means of verification", In Proc. of the 5th WSEAS/IASME Int. Conf. on Electric Power Systems, High Voltages, Electric Machines, Tenerife, Spain, December 16-18, (2005), pp 591-595

10. M. Pospisilik, J. Soldan, M. Adamek, Influence of the Properties of a Real Semi Anechoic Chamber on an Internal Electromagnetic Field Distribution, WSEAS Transactions on Systems, 14, (2015), pp 174 - 186, EISSN: 2224-2678

11. J. A. Cartrysse, "Measuring method for the characterization of shielding gaskets", in 8th International Conference on Electromagnetic Compatibility, pp 251 - 253, Heriot-Watt University, Edinburgh, UK, September (1992).

12. Z. Trnka, Theory of Electrical Engineering [Teoretická elektrotechnika]. Bratislava: SNTL Alfa, (1972)

13. V. Kus, Effects of Converters with Semiconductors to the Power System [Vliv polovodičových méničů na napájeci soustavu], Prague: BEN-Technicka literature, (2002), ISBN: 80-730-0062-8 